\title{
Neural Consequences of Competing Stimuli in Both Visual Hemifields: A Physiological Basis for Visual Extinction
}

\author{
Gereon R. Fink, MD, ${ }^{*} \dagger \ddagger$ Jon Driver, DPhil, $\$$ Chris Rorden, PhD, $\$$ Torsten Baldeweg, PhD, \\ and Raymond J. Dolan, MD*
}

\begin{abstract}
We used positron emission tomography in healthy volunteers to test hemispheric rivalry theories for normal and pathological spatial attention, which provide an influential account of contralesional extinction on bilateral stimulation after unilateral brain injury. Subjects reported visual characters presented either unilaterally or bilaterally. An extinction-like pattern was found behaviorally, with characters in one hemifield reported less accurately when competing characters appeared in the other hemifield. Differences in neural activity for unilateral minus bilateral conditions revealed greater activation of striate and extrastriate areas for stimuli presented without competing stimuli in the other hemifield. Thus, simultaneous bilateral stimulation led to a significant reduction in response by spatiotopic visual cortex contralateral to a particular stimulus. These data provide physiological support for interhemispheric rivalry in the intact human brain, and demonstrate that such competition impacts at early levels of perceptual processing.
\end{abstract}

Fink GR, Driver J, Rorden C, Baldeweg T, Dolan RJ. Neural consequences of competing stimuli in both visual hemifields: a physiological basis for visual extinction. Ann Neurol 2000;47:440-446

Kinsbourne $^{1}$ and others $^{2,3}$ have proposed a hemispheric rivalry account for spatial attention. From this viewpoint, stimulus-driven activity in one hemisphere tends to suppress that in the other, and vice versa, via callosal interactions. This account has become popular as an explanation for extinction, which is a common sign after many unilateral brain lesions, although classically it is associated with parietal damage. ${ }^{4}$ Patients with visual extinction respond appropriately to an isolated unilateral stimulus, irrespective of whether it appears in their contralesional or ipsilesional hemifield. On bilateral stimulation, however, the contralesional stimulus typically goes undetected. ${ }^{2,5-8}$ Hemispheric rivalry explains this as follows. Only the bilateral trials place the input to the lesioned hemisphere in direct competition with input to the intact hemisphere. This exacerbates the patient's deficit, because on bilateral trials the weakened hemisphere is suppressed by the intact hemisphere (which has effectively been strengthened in relative terms by the lesion).

A key assumption in this influential account is that interhemispheric rivalry, in response to bilateral visual stimulation, exists also in the normal human brain (although without the lesion-induced bias); but this has yet to be demonstrated physiologically. The critical prediction, of a reduced neural response to a lateralized visual stimulus when the other hemifield is stimulated simultaneously, is directly amenable to analysis with functional neuroimaging. We presented subjects with visual characters for verbal report, in either one or both visual hemifields (analogous to the unilateral and bilateral presentations used in clinical tests for extinction). The characters were sufficiently eccentric that they initially projected to one hemisphere alone, thus allowing a direct test for effects of interhemispheric competition within early retinotopic visual areas. Hemispheric rivalry should lead to a stronger response contralateral to a particular stimulus on unilateral than on bilateral trials, within retinotopic visual areas.

\section{Subjects and Methods}

Subjects

The 12 healthy, right-handed male volunteers (aged 20-36 years) had no history of neurological or psychiatric illness. Informed consent was obtained before participation. The study involved administration of $4.5 \mathrm{mSv}$ effective dose equivalents of radioactivity per subject. Permission to administer radioactivity was obtained from the Administration of Radioactive Substances Advisory Committee of the Department of Health (UK). The study was approved by the local
From the *Wellcome Department of Cognitive Neurology, Institute of Neurology, \$Institute of Cognitive Neuroscience, University College London, and "Division of Neuroscience, Imperial College London, London, UK; and †Neurologische Klinik, Heinrich-HeineUniversität Düsseldorf, Düsseldorf, and ‡Institut für Medizin, Forschungszentrum Jülich, Jülich, Germany.
Received Jun 1, 1999, and in revised form Nov 16. Accepted for publication Nov 16, 1999.

Address correspondence to Dr Fink, Neurologische Klinik, Heinrich-Heine Universität, Postfach 1010 07, 40001 Düsseldorf, Germany. 
ethics committee of the National Hospital for Neurology and Neurosurgery (London, UK).

\section{Behavioral Paradigm}

We measured brain activity while subjects engaged in a visual character-identification task. The four conditions all involved subjects fixating on a central cross while (in conditions 1 and 2) a column of three letters was briefly presented unilaterally, either in the left (condition 1) or right visual field (condition 2 ); or letters were presented bilaterally (in conditions 3 and 4), with three letters within each visual field simultaneously (to yield a total of six letters; a column of three letters on each side). In all conditions, the task was to name as many letters as possible. However, based on classic psychophysical findings with this whole-report task for brief letter displays, ${ }^{9,10}$ we anticipated that no more than about three letters could be reported for all conditions. Note that this normal inability to extract more than approximately three letters from a brief display is known to reflect limits in visual processing capacity, not limitations in verbal retention. ${ }^{9-11}$ For our bilateral displays, comprising six letters, the phenomenon produced is that people can easily report most of the letters from one side or the other, but cannot report all the letters from both sides together, producing an extinction-like situation. In bilateral condition 3, subjects were asked to report as many letters as possible, but starting with those on the left side (ie, just as they would for the unilateral left condition), followed by as many as possible from the right side; whereas the reverse applied for bilateral condition 4. This allowed us to test behaviorally for the following extinction-like pattern-either side being reportable on unilateral trials but only the prioritized side being reportable on bilateral trials.

Letter displays appeared on a 17 -inch computer screen, viewed from a distance of $40 \mathrm{~cm}$. A red fixation cross of $1.9^{\circ}$ appeared at the center. Letters were displayed in black against a white background (to eliminate slow phospor decay), in 136-point Times New Roman font, with a height of $9.5^{\circ}$ for each letter; 18 possible letter identities were used (omitting A, E, I, O, U, and $\mathrm{Y}$, to prevent columns from forming pronounceable strings, and $\mathrm{W}$ plus $\mathrm{M}$ because of their width). The innermost edge of the middle letter in each column of three was presented $9.9^{\circ}$ from central fixation, whereas the top and bottom letters were inset slightly $\left(8^{\circ}\right.$ from the horizontal midline) to compensate for the drop in acuity at upper and lower positions. Vertically, $4^{\circ}$ separated letters within a column. No letter identity was repeated within a single display, but letter selection was otherwise random.

Each trial began with the fixation cross flashing (off for $120 \mathrm{msec}$, on for $500 \mathrm{msec}$, then off-on-off for $100 \mathrm{msec}$ each, and finally remaining on) to remind subjects to fixate, and to warn of the upcoming letters; $200 \mathrm{msec}$ later, a letter display appeared for $200 \mathrm{msec}$ (too brief to allow saccades during its presentation). After the offset of the letters, 1,830 msec elapsed before fixation flashed again. Thus, each trial took $3,150 \mathrm{msec}$ in total, allowing time for the letters to be reported verbally between displays. Thirty trials of one condition were presented within each block.

Before the scanning session, subjects were given practice (at least two blocks of 30 trials for each condition), with feedback on fixation accuracy and letter report. The impor- tance of maintaining central fixation throughout was repeatedly emphasized, and further practice was given until each subject was able to achieve this reliably. In the scanning session, subjects were given a short practice of 10 trials at the next condition before the next scan and reminded of the importance of holding fixation. A video camera provided a visual check on the subject's eye position (in addition to formal electro-oculographic [EOG] recording) and was also used to record the subject's spoken responses for off-line scoring by a rater blind to our conditions and hypotheses.

\section{Eye Position}

Horizontal EOG was continuously recorded for 90 seconds during each scan, using a Neuroscan system (Sterling, VA). Pairs of silver/silver chloride electrodes were attached to left and right outer canthi, with the ground electrode on the middle of the forehead. Electrode impedance was kept below $5 \mathrm{k} \Omega$, which resulted in drift-free recordings (as confirmed by our calibration checks; see below) over the whole scanning period. System bandpass was 0 to $30 \mathrm{~Hz}$, with signals digitized at $100 \mathrm{~Hz}$. Because EOG signals can drift over lengthy periods, we implemented a calibration check immediately before and after each scan, with the subject making horizontal saccades to follow the fixation cross as it jumped from center to the left or right (alternately at $7^{\circ}$ or $14^{\circ}$ ) and back to the center again. The corresponding EOG deflections served as calibration for EOG data analysis and for determining the EOG reading corresponding to the subject fixating the central point.

For assessment of eye position during the letter displays, every individual EOG record (total duration of 90 seconds) was first visually inspected for systematic changes in eye position. Only 1 subject showed such changes (looking toward the side of unilateral displays) and, accordingly, was excluded from all the analyses reported below on behavioral and imaging data sets. A quantitative analysis was performed on eye position for the remaining subjects $(\mathrm{n}=11)$. To check that fixation was central when the brief letter displays were presented, we compared mean EOG when looking at central fixation during the calibration checks with mean EOG before every letter display. For this purpose, EOG was averaged over 500 -msec periods after initiation of each calibration fixation and for $500 \mathrm{msec}$ before each letter display. There were no differences between fixation calibration and any experimental condition, indicating that subjects held central fixation before each letter display in accordance with instructions.

\section{Imaging Hardware and Procedures}

Relative regional cerebral blood flow $(\mathrm{rCBF})$ was measured by recording the regional distribution of cerebral radioactivity after the intravenous injection of ${ }^{15} \mathrm{O}$-labeled water by using a Siemens/CPS Ecat Exact HR+ (Model 962) whole brain positron emission tomographic (PET) scanner (CTI, Knoxville, TN). For each relative rCBF measurement, $9 \mathrm{mCi}$ of $\mathrm{H}_{25} \mathrm{O}$ was given intravenously. Twelve consecutive emission PET scans were collected in three-dimensional mode sequentially over 90 seconds, after tracer arrival in the brain, and corrected for background activity and effects of radiation attenuation. The corrected data were reconstructed into 63 transverse planes (separation, $2.4 \mathrm{~mm}$ ) and into $128 \times 128$ 
pixels (size, $2.1 \mathrm{~mm}$ ) by three-dimensional filtered backprojection by using a Hann filter of cutoff frequency 0.5 cycles per voxel and by applying a scatter correction. The resolution of the images was $6 \mathrm{~mm}$ (at full width half maximum).

In a separate session, a magnetic resonance imaging (MRI) scan of each subject's brain was obtained to exclude the possibility of morphological abnormalities and for stereotactic normalization into the standard anatomical space (see below). The MRI scan used a 2-T system (Vision; Siemens AG, Germany) with a three-dimensional T1-weighted imaging technique to produce 108 transaxial slices $(1 \times 1 \times 1.5 \mathrm{~mm})$.

\section{Image Analysis}

Statistical parametric mapping software (SPM97d; Wellcome Department of Cognitive Neurology, London, UK; http:// www.fil.ion.ucl.ac.uk) was used for image realignment, image normalization, smoothing, and to create statistical maps of significant relative rCBF changes. ${ }^{12,13}$ PET images were filtered by using a low-pass Gaussian filter (resulting in an image resolution of $12 \mathrm{~mm}$ ) to reduce the variance due to individual anatomical variability and to improve signal-tonoise ratio. The resulting voxel size in stereotactic space was $2 \times 2 \mathrm{~mm}$ with an interplane distance of $4 \mathrm{~mm}$. Data were thereafter expressed in terms of standard stereotactic coordinates in $x, y$, and $z$ axes (as defined in Table 2). After stereotactic normalization, the effects of interest were estimated separately on a voxel-by-voxel basis by using SPM97d. ${ }^{12}$ Differences in global CBF, within and between subjects, were removed by treating global activity as the covariate. Comparisons of condition specific means were made by using the $t$ statistic and thereafter transformed into normally distributed $Z$ statistics. The resulting set of $z$ values constituted a statistical parametric map $\left(\mathrm{SPM}_{\{z\}}\right.$ map). The level of significance was set at $p<0.001$ (uncorrected for multiple comparisons ${ }^{14}$ ) for areas within striate and extrastriate cortex, because for these spatiotopic visual areas we had clear a priori predictions about which unilateral stimuli (ie, left versus right) should activate which hemisphere, based on the laterality of their cortical projections. ${ }^{15,16}$ Activations outside these early visual areas are only reported at $p<0.05$, corrected for multiple comparisons, because we could have no analogous predictions for brain areas that might respond directly to stimuli in either visual field.

The stereotactic coordinates for local maxima within areas of significant relative rCBF change were determined. ${ }^{12}$ The anatomical localization of these was assessed by reference to the standard stereotactic atlas, ${ }^{17}$ and validation of this was obtained by superimposition of the $\operatorname{SPM}_{\{z\}}$ maps on the group mean MRI scan calculated after each individual's MRI scan had been stereotactically transformed into the same standard stereotactic space.

\section{Results}

\section{Behavioral Measures}

The mean accuracy of the letter report for the accepted subjects $(\mathrm{n}=11)$ in each condition is shown in Table 1. Overall, $88 \%$ of unilateral letters were named correctly. By contrast, only $13 \%$ of letters on the nonprioritized side were reported during bilateral trials, a de-
Table 1. Percentage of Correctly Reported Letters for Each Side in Each Condition

\begin{tabular}{llll}
\hline $\begin{array}{l}\text { Side of } \\
\text { Scored Letters }\end{array}$ & $\begin{array}{l}\text { Type of } \\
\text { Stimulation }\end{array}$ & $\begin{array}{l}\text { Side Reported } \\
\text { First }\end{array}$ & $\begin{array}{l}\text { Correct } \\
\text { Responses (\%) }\end{array}$ \\
\hline \multirow{2}{*}{ Left } & Unilateral & L & 86 \\
& Bilateral & L & 79 \\
\multirow{4}{*}{ Right } & Bilateral & R & 15 \\
& Unilateral & R & 89 \\
& Bilateral & R & 80 \\
& Bilateral & L & 10 \\
\hline
\end{tabular}

cline that was highly reliable $[F(1,10)=1,284.0 ; p<$ $0.000001]$. The prioritized side on bilateral trials also showed a tendency to suffer from presentation of simultaneous competitors on the other side $(80 \%$ correct vs $88 \%$ for unilateral trials), but this was not significant. Thus, although subjects reported most letters correctly on unilateral trials, they missed most of these same letters when on the nonprioritized side under bilateral stimulation, thus producing an extinction-like pattern. This limit in normal capacity has been well documented previously, and reflects specifically visual rather than verbal limitations. ${ }^{9-11}$

The overall number of verbal responses made (ie, correct plus incorrect responses) did not differ reliably between the four conditions; hence, comparing the visual conditions should not be confounded with any verbal differences. The average number of words spoken across the whole experiment was 257 for condition 1, 257 for condition 2, 260 for condition 3, and 276 for condition 4. The slight tendency for more responses in the latter condition (bilateral display, select right side first) did not approach significance.

\section{PET Activations}

We first examined the simple effects of unilateral stimulation in different hemifields, which should activate retinotopic visual areas contralateral to the stimulated field. ${ }^{15,16}$ Comparison of conditions 1 and 2 (ie, unilateral left minus unilateral right, and vice versa) revealed, as expected, greater neural activity in early visual areas within the occipital and temporo-occipital cortex, contralateral to the stimulation (Fig, a; Table 2). The activation was somewhat more widespread for unilateral right stimulation, but the maxima of differential neural responses during the unilateral conditions were fairly symmetrical, with no differential occipital or temporo-occipital areas implied. The areas activated included those previously described as V1, V2, and V3. ${ }^{15,16}$

To assess whether these contralateral neural responses to lateralized stimulation were reduced during simultaneous bilateral stimulation, as hemispheric rivalry predicts, we compared unilateral and bilateral 

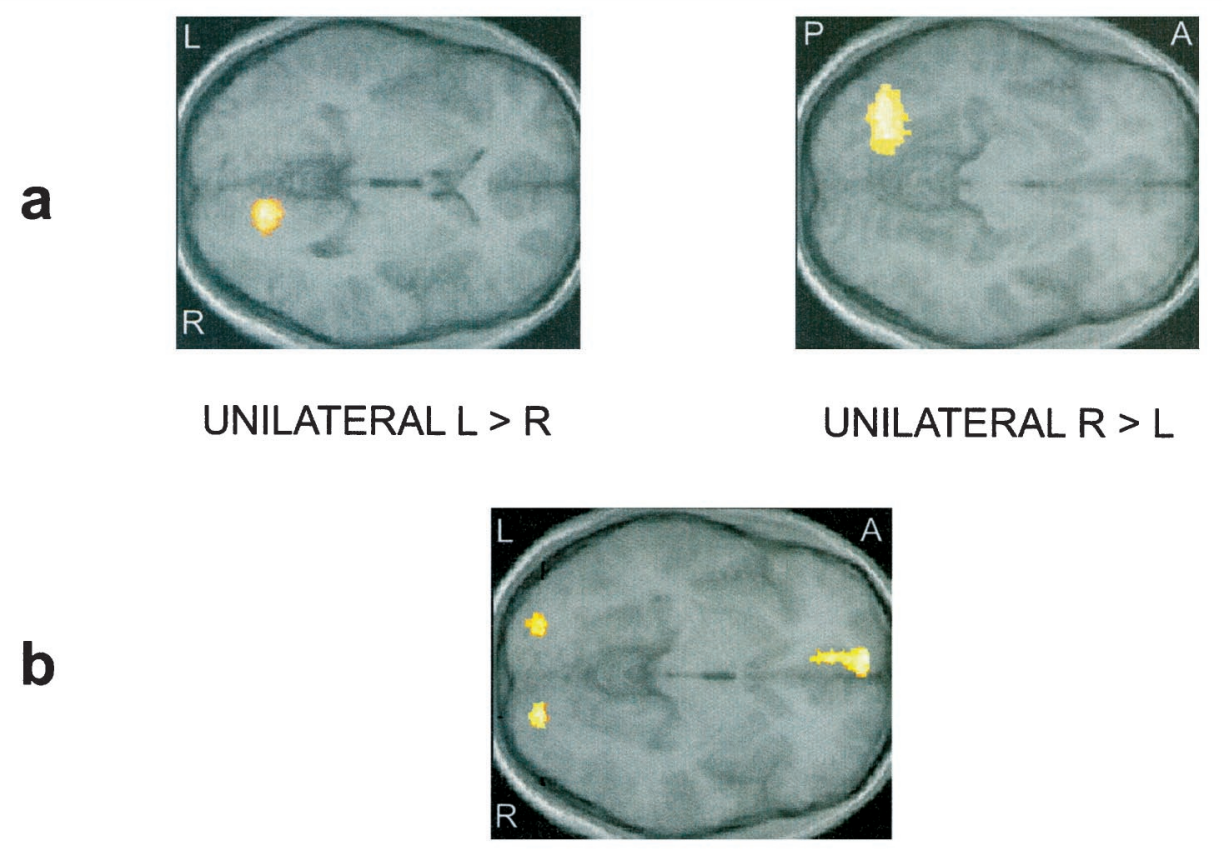

\section{UNILATERAL > BILATERAL}

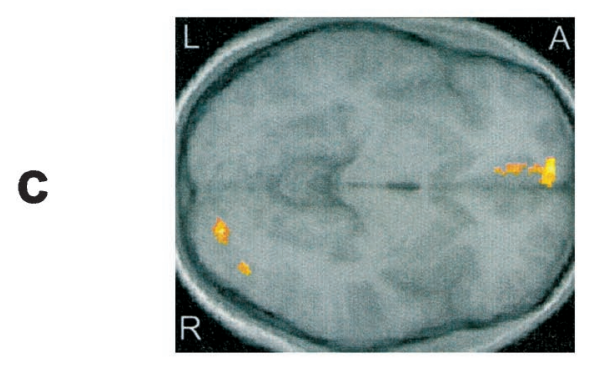

UNILATERAL L > BILATERAL

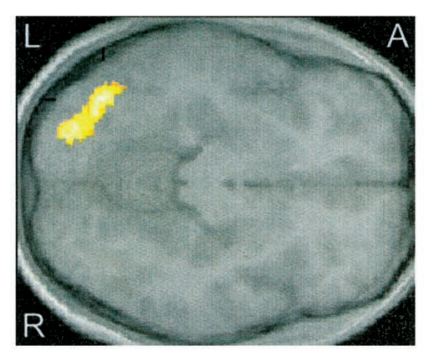

UNILATERAL R > BILATERAL

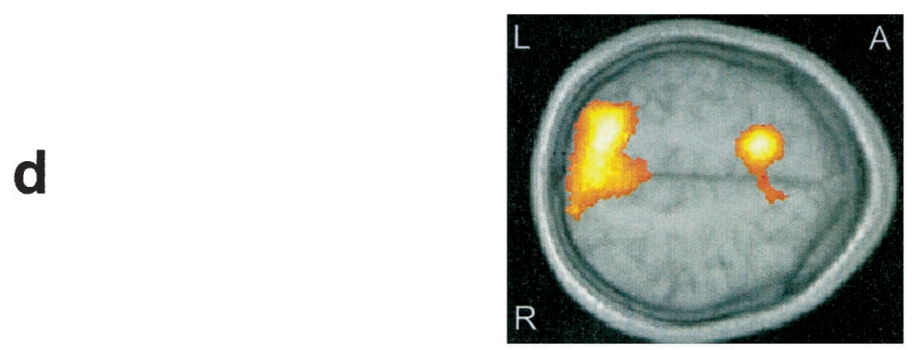

BILATERAL > UNILATERAL

Fig. Relative regional cerebral blood flow ( $r C B F$ ) increases (for the 11 subjects) associated with unilateral left $>$ unilateral right (condition $1-$ condition 2) (a, left) and unilateral right $>$ unilateral left (condition $2-$ condition 1$)(a$, right), unilateral $>$ bilateral (conditions $(1+2)-(3+4)](b)$, unilateral left $>$ bilateral (conditions $1-3-4)(c$, left) and unilateral right $>$ bilateral (conditions $2-3-4)$ (c, right), and bilateral $>$ unilateral (conditions $3+4-1-2)$ (d). Areas of significant relative $r C B F$ increase $(\mathrm{p}<0.001$, uncorrected) are shown as transverse sections through the local maxima within the regions activated (see Tables 2 and 3). Transverse $S P M_{\{z\}}$ maps are superimposed on the group mean magnetic resonance imaging scan, which had been spatially normalized into the same anatomical space. The exact coordinates of the local maxima within the regions activated and their $\mathrm{Z}$ statistics are given in Tables 2 and 3. $\mathrm{L}=$ left; $\mathrm{R}=$ right; $\mathrm{A}=$ anterior; $\mathrm{P}=$ posterior. Note that among the activations shown, only the left frontal cluster for unilateral $>$ bilateral and unilateral $L>$ bilateral failed to reach the criteria for significance (see Subjects and Methods). 


\begin{tabular}{|c|c|c|c|c|c|}
\hline \multirow[b]{2}{*}{ Region } & \multirow[b]{2}{*}{ Side } & \multicolumn{3}{|c|}{ Coordinates } & \multirow[b]{2}{*}{$Z$ Score } \\
\hline & & $x$ & $y$ & $z$ & \\
\hline \multicolumn{6}{|l|}{ Unilateral left $>$ unilateral right } \\
\hline $\begin{array}{l}\text { Primary visual cortex } \\
\text { (BA 17) }\end{array}$ & $\mathrm{R}$ & 14 & -72 & 0 & 4.9 \\
\hline $\begin{array}{l}\text { Lingual gyrus } \\
\quad \text { (BA 18) }\end{array}$ & $\mathrm{R}$ & 20 & -68 & -10 & 3.8 \\
\hline $\begin{array}{l}\text { Medial/superior occipital gyrus } \\
\quad \text { (BA 18/19) }\end{array}$ & $\mathrm{R}$ & 20 & -88 & 22 & 3.8 \\
\hline \multicolumn{6}{|l|}{ Unilateral right $>$ unilateral left } \\
\hline $\begin{array}{l}\text { Medial/superior occipital gyrus } \\
\text { (BA 18/19) }\end{array}$ & $\mathrm{L}$ & -8 & -92 & 24 & 6.1 \\
\hline $\begin{array}{l}\text { Superior occipital gyrus } \\
\text { (BA 19) }\end{array}$ & $\mathrm{L}$ & -14 & -86 & 26 & 5.2 \\
\hline $\begin{array}{l}\text { Primary visual cortex } \\
\quad \text { (BA 17) }\end{array}$ & $\mathrm{L}$ & -4 & -94 & 12 & 4.4 \\
\hline $\begin{array}{l}\text { Fusiform gyrus } \\
\text { (BA } 18 / 19)\end{array}$ & $\mathrm{L}$ & -40 & -72 & -12 & 5.3 \\
\hline $\begin{array}{l}\text { Inferior occipital gyrus } \\
\quad \text { (BA 18/19) }\end{array}$ & $\mathrm{L}$ & -32 & -74 & -6 & 5.3 \\
\hline $\begin{array}{l}\text { Lingual gyrus } \\
\quad(\text { BA 18) }\end{array}$ & $\mathrm{L}$ & -20 & -70 & -8 & 5.3 \\
\hline
\end{tabular}

Coordinates in standard stereotactic space ${ }^{17}$ refer to maximally activated foci as indicated by the highest $Z$ score within an area of activation associated with unilateral presentation of stimuli in the left visual hemifield or unilateral presentation of stimuli in the right visual hemifield.

$x=$ distance $(\mathrm{mm})$ to right $(+)$ or left $(-)$ of the midsagittal (interhemispheric) line; $y=$ distance anterior $(+)$ or posterior $(-)$ to vertical plane through the anterior commissure; $z=$ distance above $(+)$ or below $(-)$ the intercommissural line. For each location an estimate of the Brodmann area (BA) is given in parentheses, which is based on the stereotactic atlas ${ }^{17}$ and group mean magnetic resonance imaging scan after normalization into standard stereotactic space (see Subjects and Methods).

conditions overall [both unilaterals minus both bilaterals: conditions $(1+2)>(3+4)]$. Note that for this main effect, unilateral stimulation of both visual fields is considered, thus allowing an appropriate comparison with the bilateral conditions. This analysis revealed (Table 3; see Fig, b) more neural activity in response to unilateral stimulation than bilateral stimulation, in posterior visual areas. The local maxima of the activated areas lay within areas V2 and V3, but the activations also include primary visual cortex as previously defined. ${ }^{15,16}$ Although some activity was also seen in a frontal cluster, this failed to reach significance.

The higher activation for unilateral conditions in posterior visual areas is consistent with the hemispheric rivalry prediction of mutual suppression between hemifields. The rivalry account specifically suggests that the left hemisphere response to right unilateral stimulation should be reduced during bilateral stimulation (and likewise for the right hemisphere response to left unilateral stimulation). This specificity was examined by testing for regions that were more active during each individual unilateral condition, versus the bilateral conditions. Comparison of right unilateral stimulation (condition 2) minus bilateral stimulation (conditions 3 and 4) revealed left hemisphere occipital and temporooccipital activations in fusiform and lingual gyri, extending into primary visual cortex (see Table 3 and
Fig, c, right). The equivalent comparison of left unilateral minus bilateral showed a similar (albeit less extensive) pattern of activations in the right hemisphere (see Table 3 and Fig, c, left).

Finally, for completeness, the main effect of both bilateral conditions minus both unilaterals was assessed, to determine any areas activated more by bilateral than unilateral stimulation (note that unilateral stimulation of both visual fields is considered in this comparison). This revealed (see Table 3 and Fig, d) bilateral activation of the superior posterior parietal cortex (Brodmann area 7), extending laterally into more inferior aspects of the posterior parietal cortex (Brodmann area 40). Dorsal aspects of the anterior cingulate (Brodmann area 31/32) were also activated in the left hemisphere.

\section{Discussion}

These results provide the first direct physiological evidence for interhemispheric rivalry in the intact human brain, during bilateral visual stimulation. Presenting visual stimuli in both hemifields simultaneously reduced neural activity in early visual areas, relative to that found contralateral to a unilateral stimulus. The seemingly paradoxical prediction of hemispheric rivalry accounts, ${ }^{3,18}$ that fewer stimuli can lead to more activity, was thus confirmed. Because our stimuli were presented in peripheral vision, and the activations were 


\begin{tabular}{|c|c|c|c|c|c|}
\hline \multirow[b]{2}{*}{ Region } & \multirow[b]{2}{*}{ Side } & \multicolumn{3}{|c|}{ Coordinates } & \multirow[b]{2}{*}{$Z$ Score } \\
\hline & & $x$ & $y$ & $z$ & \\
\hline \multicolumn{6}{|l|}{ Unilateral (left and right) $>$ bilateral } \\
\hline Lingual gyrus/fusiform gyrus & $\mathrm{L}$ & -24 & -90 & -10 & 3.9 \\
\hline (BA 18/19) & $\mathrm{R}$ & 20 & -90 & -4 & 3.8 \\
\hline \multicolumn{6}{|l|}{ Unilateral right $>$ bilateral } \\
\hline Fusiform gyrus & $\mathrm{L}$ & -40 & -72 & -10 & 4.9 \\
\hline (BA 18/19) & $\mathrm{L}$ & -26 & -84 & -10 & 4.5 \\
\hline & $\mathrm{L}$ & -32 & -74 & -6 & 4.4 \\
\hline \multicolumn{6}{|l|}{ Unilateral left $>$ bilateral } \\
\hline $\begin{array}{l}\text { Fusiform gyrus } \\
\text { (BA 18/19) }\end{array}$ & $\mathrm{R}$ & 44 & -78 & -10 & 3.5 \\
\hline $\begin{array}{l}\text { Lingual gyrus } \\
\text { (BA 18/19) }\end{array}$ & $\mathrm{R}$ & 20 & -92 & -4 & 3.0 \\
\hline \multicolumn{6}{|l|}{ Bilateral $>$ unilateral } \\
\hline \multirow{5}{*}{$\begin{array}{l}\text { Superior posterior parietal cortex } \\
\text { (BA 7) }\end{array}$} & $\mathrm{L}$ & -16 & -68 & 54 & 5.4 \\
\hline & $\mathrm{L}$ & -22 & -62 & 52 & 5.2 \\
\hline & $\mathrm{L}$ & -30 & -64 & 54 & 4.7 \\
\hline & $\mathrm{R}$ & 4 & -62 & 58 & 4.3 \\
\hline & $\mathrm{R}$ & 6 & -76 & 52 & 4.1 \\
\hline $\begin{array}{l}\text { Anterior cingulate cortex } \\
\text { (BA 31/32) }\end{array}$ & $\mathrm{L}$ & -16 & 8 & 52 & 5.2 \\
\hline
\end{tabular}

For details, see Table 2.

lateralized within early visual areas, competitive interhemispheric interactions (presumably across the callosum) provide the most likely mechanism for the observed effects. The greater activation for unilateral than bilateral presentations cannot be explained by suggesting that the activated areas respond directly to both hemifields. This is implausible given the anatomical coordinates, plus the simple effects observed for left versus right unilateral stimulation (see Fig, a, and Table 2 ); and, in any case, it would be expected to produce the opposite result of stronger activation for bilateral displays. Our results, accordingly, suggest that hemispheric rivalry can arise at early stages of sensory processing; indeed, even primary visual cortex appears to show the effect. Given that callosal connections are not thought to operate for the peripheral field in this particular brain region, it seems possible that the modulation of primary visual cortex reflects feedback from the subsequent retinotopic visual areas that were activated, for which callosal connections between homologous peripheral locations in each hemifield are known to exist in primates. ${ }^{19}$

Our findings of competitive suppression with bilateral stimulation appear consistent with several previous results and also extend them. A recent functional MRI study ${ }^{20}$ found that activation in presumed V4 for an isolated visual stimulus, presented centrally just above the horizontal meridian, was reduced when competing stimuli were presented just below this meridian. As for our own results, these show that competing stimuli can reduce the visual response to an individual stimulus.
However, the previous study ${ }^{20}$ could not test for interhemispheric rivalry, because all stimuli were projected to the same hemisphere. Moreover, the competing stimuli in this previous study were extremely close (almost abutting), whereas those in our study were at least $16^{\circ}$ apart, and in separate hemifields, yet mutual suppression was still found.

A study with transcranial magnetic stimulation produced some previous data in support of hemispheric rivalry, ${ }^{21}$ by documenting that transcranial magnetic stimulation at right parietal sites reduces threshold for detecting tactile stimuli on the right (ipsilateral) thumb. This result was taken to show that disabling the right hemisphere releases the left hemisphere from rivalrous inhibition. However, other interpretations for that study are possible (because right hand sensitivity was not measured, nor was the effect of transcranial magnetic stimulation over the left hemisphere). Moreover, it differs from the present experiment not only in sensory modality, but also in relying on a behavioral dependent measure, rather than a direct measure of neural activity as here.

Our predictions focused on contralateral visual areas showing greater activation for unilateral than bilateral presentation; these were confirmed for striate and extrastriate areas. However, some brain regions showed the opposite pattern (for which the rivalry model makes no specific anatomical predictions) of more activity for bilateral displays than for left plus right unilateral displays. The site of these activations is interesting in relation to clinical phenomena. The pattern of 
bilateral superior posterior parietal activations, extending posteriorly to the parieto-occipital junction, are reminiscent of the distribution of bilateral lesions associated with "simultanagnosia," $22-24$ a condition characterized by an inability to see multiple objects concurrently. Note that this activation was specifically found in a condition where the task required normal subjects to see more concurrent objects (six rather than three). The anterior cingulate (Brodmann area 31/32) activation may similarly reflect the increased attentional demands ${ }^{25}$ when reporting stimuli from both visual hemifields.

In conclusion, our findings provide physiological support for interhemispheric competition during bilateral visual stimulation in the intact human brain. Our results suggest that such competition may impact at early levels of visual processing. The stronger activation resulting from unilateral than bilateral stimuli mirrored an extinction-like pattern in the behavioral data for our healthy subjects. Pathological extinction ${ }^{2,5-8}$ after unilateral brain injury may reflect an imbalance in this interhemispheric competition, which particularly disadvantages the contralesional field during bilateral stimulation.

This study was supported by the Wellcome Trust, plus additional support from the Medical Research Council (UK) to Dr Driver. Dr Fink is supported by the Deutsche Forschungsgemeinschaft (SFB 194, TP A16).

We thank our volunteers, plus the radiography and technical staff at the Wellcome Department.

\section{References}

1. Kinsbourne M. Hemineglect and hemisphere rivalry. Adv Neurol 1977;18:41-49

2. Cohen JC, Farah MJ, Romero RD, Servan-Schreiber D. Mechanisms of spatial attention. J Cogn Neurosci 1994;6:377-387

3. Driver J, Mattingley JB, Rorden C, Davis G. Extinction as a paradigm measure of attentional bias and restricted capacity after brain injury. In: Thier $\mathrm{P}$, Karnath $\mathrm{H}-\mathrm{O}$, eds. Parietal lobe contributions to orientation in 3D space. Heidelberg: Springer, 1997:401-430

4. Vallar G, Rusconi ML, Bignamini L, et al. Anatomical correlates of visual and tactile extinction in humans: a clinical CT scan study. J Neurol Neurosurg Psychiatry 1994;57:464-470

5. Bender MB. Disorders in perception. Springfield, IL: Charles C Thomas, 1952
6. Loeb J. Die elementaren Störungen einfacher Funktionen nach oberflächlicher umschriebener Verletzung des Großhirns. Pflügers Arch Physiol 1885;37:51-56

7. Oppenheim H. Uber eine durch eine klinisch bisher nicht verwertete Untersuchungsmethode ermittelte Sensibilitätsstörung bei einseitigen Erkrankungen des Großhirns. Neurologisches Centralblatt 1885;4:529-533

8. Poppelreuter W. Die psychischen Schädigungen durch Kopfschuß im Kriege 1914/16. Leipzig: Leopold Voss, 1917

9. Duncan J. The locus of interference in the perception of simultaneous stimuli. Psychol Rev 1980;87:272-300

10. Sperling G. The information available in brief visual presentations. Psychol Monogr 1960;74:1-29

11. Bundesen C. A theory of visual attention. Psychol Rev 1990; 97:523-547

12. Friston KJ, Holmes A, Worsley KJ, et al. Statistical parametric maps in functional imaging: a general linear approach. Hum Brain Mapp 1995;2:189-210

13. Friston KJ, Ashburner J, Frith CD, et al. Spatial registration and normalization of images. Hum Brain Mapp 1995;3:165189

14. Friston KJ. Testing for anatomically specified regional effects. Hum Brain Mapp 1997;5:133-166

15. Sereno MI, Dale A, Reppas JB, et al. Borders of multiple visual areas in humans revealed by functional magnetic resonance imaging. Science 1995;268:889-893

16. Shipp S, Watson JDG, Frackowiak RSJ, Zeki S. Retinotopic maps in human prestriate visual cortex: the demarcation of areas V2 and V3. Neuroimage 1995;2:125-132

17. Talairach J, Tournoux P. Co-planar stereotactic atlas of the human brain. Stuttgart: Thieme, 1988

18. Kinsbourne M. The cerebral basis of lateral asymmetries in attention. Acta Psychol 1970;33:193-201

19. Zeki S. A vision of the brain. Oxford: Blackwell Scientific, 1993

20. Kastner S, De Weerd P, Desimone R, Ungerleider LG. Mechanisms of directed attention in the human extrastriate cortex as revealed by functional MRI. Science 1998;282:108-111

21. Seyal M, Ro T, Rafal R. Perception of subthreshold cutaneous stimuli following transcranial magnetic stimulation of ipsilateral parietal cortex. Ann Neurol 1995;38:264-267

22. Balint R. Seelenlähmung des Schauens, optische Ataxie, räumliche Störung der Aufmerksamkeit. Monatsschrift für Psychiatrie und Neurologie. 1909;25:51-81

23. Friedman-Hill SR, Robertson LC, Treisman A. Parietal contributions to visual feature binding: evidence from a patient with bilateral lesions. Science 1995;269:853-855

24. Rafal R, Robertson LC. The neurology of visual attention. In: Gazzaniga MS, ed. The cognitive neurosciences. Cambridge, MA: MIT Press, 1995:625-648

25. Carter CS, Braver TS, Barch DM, et al. Anterior cingulate cortex, error detection, and the online monitoring of performance. Science 1998;280:747-749 